\title{
Existentielle Angst und ihre Verkörperung
}

\author{
Christian Tewes
}

\section{Einleitung}

Eine Untersuchung der Frage, wie sich die Angst individuell und sozial verkörpert - also insbesondere auch leiblich realisiert - sieht sich im Rahmen einer Philosophie der Gefühle verschiedenen Fragen gegenüber. So haben kognitivistische Emotionstheoretiker gegen die in der Tradition von William James vertretene Auffassung, dass die Empfindung von Emotionen im phänomenalen Bewusstsein eigener veränderter körperlicher Zustände bestünde, ${ }^{1}$ eingewandt, dass dabei bedeutende Dimensionen von Emotionen unerklärbar bleiben. ${ }^{2}$ Ein zentraler Einwand betrifft hier die Intentionalität bzw. Gerichtetheit von Emotionen. Wenn eine Person Angst hat, einen Raum zu betreten, in dem die Aufmerksamkeit auf sie gerichtet ist - wie bei sozialen Phobien - dann ist die Angst auf ein Objekt oder Szenario der Außenwelt gerichtet. Wie Robert C. Roberts als bedeutender Vertreter des kognitiven Ansatzes ausgeführt hat, sind Emotionen wie Angst aus diesem Grund immer auch als Propositionen formulierbar. ${ }^{3}$ Dass Emotionen mit körperlichen Veränderungen einhergehen, möchte er dabei keinesfalls leugnen. Anders als frühere Kognitivisten setzt er Emotionen deshalb auch nicht mit Urteilen gleich. Doch sieht er in körperlichen Begleiterscheinungen wie bei der Angst lediglich Symptome, auf die der emotionale Fokus eben in der Regel nicht gerichtet sei.

Diese körperlichen Veränderungen als eine bloße Begleiterscheinung von Emotionen zu charakterisieren, greift jedoch offensichtlich zu kurz. Denn die unterschiedlichen Facetten der Angst haben

\footnotetext{
1 Sabine Döring, Allgemeine Einleitung: Philosophie der Gefühle heute, in: Philosophie der Gefühle, hg. v. Sabine Döring, Frankfurt 2009, 12-65, hier 20.

2 Anders als z.B. Antonio Damasio in seine neurowissenschaftlichen Arbeiten verwende ich die Ausdrücke "Emotionen" und "Gefühle» in diesem Aufsatz synonym. Beide Ausdrücke verwende ich dabei so, dass sie sowohl einen Bezug zum fühlenden Subjekt als auch zur Lebenswelt haben, wobei diese Relation noch einmal in eine intentionale und existentielle Bezugnahme im Rahmen des Textes ausdifferenziert wird.

3 Robert C. Robert, Was eine Emotion ist: eine Skizze, in: Philosophie der Gefühle, hg. v. Sabine Döring, 169-201, hier 184, u. 197ff.
} 
dabei mindestens einen zweifachen Charakter: Auf der einen Seite gehen Angstzustände mit konkreten physiologischen Veränderungen einher, die ein Mediziner aufgrund einer veränderten Muskelanspannung, Blutzirkulation oder bestimmten neuronalen Vorgängen wie im limbischen System messen kann. Auch eine evolutionär biologische Funktion, die sich bereits im Entdecken von Gefahren und entsprechenden Fluchtreflexen manifestiert, lässt sich dem Angstverhalten zuordnen. Auf der anderen Seite gibt es aber auch unleugbare phänomenale Dimensionen, die mit derartigen biologischphysiologischen Faktoren einhergehen. Die Gefühle der Beengung oder auch Beklemmung, die mit der Angst verbunden sind, bis hin zu einer schockartigen Lähmung oder einer panikartigen Reaktion deuten auf unterschiedliche erlebte Facetten der Angst hin. Sie stehen auch nicht nur zufällig mit den genannten physiologischen Veränderungen und Verhaltensweisen in Verbindung, sondern können sie auch teilweise mit erklären, so wie etwa Fluchtverhalten aus einem überfüllten Raum nach einer heftigen Panikattacke verstehbar wird.

In den sogenannten kognitiv-affektiven Neurowissenschaften lässt sich hingegen eine andere, nämlich internalistische, Tendenz erkennen, Gefühle zu bestimmen. Körperliche Erregungen werden im Gehirn als Affekte bzw. Gefühle bestimmt und es wird davon ausgegangen, dass die vollständigen neuronalen Erklärungen dieser Vorgänge auch eine vollständige Erklärung von Emotionen wie der Angst liefern werde. Es soll im Rahmen dieses Aufsatzes aufgezeigt werden, dass der enaktiv-verkörperungstheoretische Ansatz, wie er in jüngerer Zeit zum Beispiel für die Emotionsforschung von Giovanna Colombetti weiter entwickelt worden ist, einen alternativen Forschungsrahmen zu Gefühlen wie zur Angst zu begründen vermag: ${ }^{4}$ Einerseits möchte dieser Ansatz der konkreten Mit- und Umweltbezogenheit von Emotionen bzw. ihrer konkreten situativen Einbettung in unterschiedlichen Handlungskotexten gerecht werden. Andererseits ist zu untersuchen, ob die Verkörperungstheorie der Kognition auch in der Lage ist, die körperlich-leibliche Fundierung der Angst als bedeutendes Moment ihrer Realisierung auszuzeichnen, ohne sie jedoch unter Absehung des Umweltbezuges darauf zu reduzieren.

Es ist das Ziel, diese Gesichtspunkte im Hinblick auf das existentielle Gefühl der Angst in Anlehnung an Matthew Ratcliffes Theorie existentieller Gefühle aufzuzeigen. Denn was diesen Gefühlen fehlt, ist die konkrete intentionale Gerichtetheit auf Objekte oder Situa-

4 Giovanna Colombetti, The feeling body: affective science meets the enactive mind, Cambridge 2014. 
tionen wie dies andere Gefühle auszeichnet. Trotzdem haben selbst diese Gefühle, so die These, einen auf ihre Um- und Mitwelt bezogenen Gehalt. Sie sind deshalb prädestiniert dazu, die Verschränkung der Selbst- und Weltbezüglichkeit der Gefühle aufzuzeigen. Damit ist gemeint, dass jeder Selbstbezug auch durch einen emotional bestimmten Umweltbezug mit-konstituiert ist und umgekehrt.

In einem ersten Schritt wird zu diesem Zweck zunächst im Hinblick auf die Angst als existentiellem Gefühl ausgeführt, inwiefern sie sowohl welthaltig als auch auf unterschiedlichen Erklärungsebenen selbstbezüglich sein kann. Daraufhin führe ich aus, wie der verkörperungstheoretische Ansatz zu einer Erforschung der leiblichen aber auch kulturellen Realisierung der Angst beitragen kann. Abschließend wird ein kurzer Ausblick gegeben, wie dieser Theorieansatz zur weiteren Erforschung der existentiellen Angst beitragen kann.

\section{Angst als existentielles Gefühl}

Existentielle Gefühle stehen in den letzten Jahren verstärkt im Fokus der Forschung. Matthew Ratcliffe hat diesen Begriff in Anlehnung an Heideggers Ausführungen zur Stimmung und Befindlichkeit in Sein und Zeit neu geprägt, um damit auf einen Phänomenbereich hinzuweisen, der in der Debatte um Emotionen häufig vernachlässigt wird. Die Welt kann manchmal als vollkommen fremdartig, irreal oder auch als distanziert erscheinen, so als wäre ich von allen Beziehungen entfremdet oder gar abgeschnitten. Umgekehrt gibt es auch eine vorherrschende grundlegende Vertrautheit mit den Dingen, Personen und Situationen, sodass man sich mit ihnen und der Welt als vereint erlebt. Konkrete Erfahrungen, auf die wir intentional gerichtet sind, werden aufgrund solcher sich im präreflexiven Hintergrund befindenden existentiellen Gefühle - wie z.B. beim Vertrauen, dass wir uns auf Absprachen verlassen können oder Institutionen ihre Funktionen erfüllen, - überhaupt erst ermöglicht. ${ }^{5}$ Dies bedeutet mit Ratcliffes Worten: "Ways of finding oneself in the world are presupposed spaces of experiential possibility, which shape the various ways in which things can be experienced."

Dies wird besonders deutlich, wenn man, wie im sensomotorischen Enaktivismus üblich, die unauflösliche Verschränkung von

\footnotetext{
5 Matthew Ratcliffe, The feeling of being, in: Journal of Consciousness Studies 12 (8-10) (2005), 43-60, hier 45.
} 
Handeln und Wahrnehmen weiter untersucht. ${ }^{6}$ Leibliche Bewegungen fundieren die auftretenden Perzeptionen. Diese sind jedoch aufgrund ihrer Handlungsrelevanz für simultane und zukünftige Bewegungsabläufe mit-konstitutiv.

Die Wahrnehmung wird im Enaktivismus deshalb auch nicht als Repräsentation einer subjektunabhängigen Außenwelt verstanden, sondern die Tätigkeit des Subjekts wie in der konkreten leiblichen Haltung zum Raum ist der Wahrnehmung in ihrer jeweils perspektivischen Beziehung zum Subjekt »eingeschrieben". Zur weiteren Erschließung dieses Zusammenhanges spielen Gefühle eine konstitutive Rolle. Existentielle Gefühle, die auch als Stimmungen beschrieben werden können, ermöglichen und bestimmen die wechselseitige Realisierung von Wahrnehmung und Handlung dabei im hohen Maße. Denn auf der Grundlage unterschiedlicher existentieller Gefühle wird der subjektive Erfahrungsraum nicht nur anders erlebt bzw. mit anderen evaluativen Eigenschaften und strukturellen Relationen wahrgenommen. Vielmehr zeigt sich in der näheren Analyse, dass den möglichen Erfahrungsräumen, die auf der Grundlage unterschiedlicher existentieller Gefühle erschlossen werden, auch ganz unterschiedliche modale Handlungsräume korrespondieren. Folgender Gesichtspunkt mag dies veranschaulichen.

Wenn die Angst als existentielles Gefühl bzw. Stimmung auftritt, dann ist sie zunächst nicht intentional auf einen konkreten bedrohlichen Gegenstand gerichtet, wie im Gegensatz dazu bei einem sich nähernden zähnefletschenden Hund. ${ }^{7}$ Bei einer besonders affektiven Intensität kann dies zu einer Erstarrung (Schockstarre) des Bedrohten, zu einem starken Fluchtimpuls wie auch zu einem verzweifelten Angriff führen, je nachdem wie diese objektbezogene Angst in die gesamte kognitiv-volitionale Architektur des Akteurs eingebunden ist.

Fehlt der Angst ein solcher konkreter Objekt- oder Situationsbezug, dann verändert sich die Beziehung auf den gesamten Umraum des Subjekts fundamental, wie folgende Ausführungen verdeutlichen:

[W]enn sich der Angst kein definierter Gegenstand als Wovor der Flucht anbietet, generalisiert sie die Bedrohlichkeit auf die gesamte Umwelt. Die Relevanzstruktur des psychischen Feldes

6 Alva Noë, Action in perception, Cambridge/Massachusetts, /London 2004.

7 Die Unterscheidung zwischen einer gegenstandsorientierten Furcht und einer gegenstandslosen Angst wird häufig auf Søren Kirgegaards ("Der Begriff Angst") zurückgeführt. Eine Diskussion, ob diese Zuschreibung zutreffend ist, findet sich in Christoph Demmerling/Hilde Landwehr, Philosophie der Gefühle.Von Achtung bis Zorn, Stuttgart/Weimar 2007, hier 83ff. 
ist aufs Äußerste nivelliert: Einzig das Drohende oder Schreckliche bietet sich der Wahrnehmung an und beansprucht alle Aufmerksamkeit. ${ }^{8}$

Die im Zitat angesprochene "Generalisierung der Angst « liegt entweder bereits vor (bestimmt den gesamten Erfahrungsraum) oder stellt sich zunächst implizit im Erleben ein. Berühmt ist in dieser Hinsicht der Topos des dunklen Waldes, der als bedrohlich-unheimliches Szenario den subjektiven Erlebnisraum als Ganzes erfasst, ohne dass es einen konkreten Anlass für die subjektiv empfundene, schleichende Angst und Bedrohung geben müsste. Schatten, schemenhafte Gestaltformationen und nicht direkt zuzuordnende Geräusche aktualisieren und verdichten diese Stimmung der existentiellen Angst, die sich, wie im Zitat erläutert, auf die gesamte Umwelt bezieht.

Deutlich wird an diesem Beispiel gleichfalls, dass die z.B. auf Martin Heidegger zurückgehenden Unterscheidung in Sein und Zeit zwischen der Stimmung der Angst als "Modus der Befindlichkeit" - die Ratcliffes Analyse existentiellen Gefühls mit zugrunde liegt und der gegenstandsorientierten Furcht, sich in einem komplexen Wechselverhältnis befinden.

Ist eine zunächst unmerkliche existentielle Angst die Bedingung der Möglichkeit der Erfahrung des dunklen, bedrohlichen Waldes als subtiles und nicht weiter auffälliges Hintergrundgefühl, so dient das affektiv als Furcht erlebte Geräusch eines knickenden Astes oder der Ruf der Eule als Realisierung und Verstärkung dieses Erfahrungsund Handlungsraums. So kann das Oszillieren zwischen Angst und Furcht einerseits den Fokus wiederum auf die Gegenstandsseite verschieben und $\mathrm{zu}$ einer Fluchtbewegung aufgrund eines "furchterregenden Ereignisses« (Eulenruf, das Knacken im Gehölz) führen. Andererseits ist es sicherlich plausibel davon auszugehen, dass als furchterregend empfundene Ereignisse als Auslöser für ein existentielles Gefühl wie das der Angst dienen können, wobei sich letztere dadurch auszuzeichnen, dass sie in der Regel über längere Zeiträume stabil bleiben. ${ }^{9}$

Bedeutsam ist für unseren emotionstheoretischen Zusammenhang, dass existentielle Gefühle leibliche, subjektive und umweltbezogene Aspekte unauflöslich integrieren und dadurch einen bedeutsamen Beitrag zur Aufklärung derVerkörperung von Emotionen leisten. So zeigt die Analyse existentieller Gefühle wie der Angst auf, warum

\footnotetext{
8 Thomas Fuchs/Stefano Micali, Phänomenologie der Angst, in: Angst. Ein interdisziplinäres Handbuch, hg. v. Lars Koch, Stuttgart/Weimar 2013, 61-71, hier 52.

9 Jan Slaby/Achim Stephan, Affective intentionality and self-consciousness, in: Consciousness and Cognition 17 (2008), 506-513.
} 
eine Definition der Emotionen als Urteile mit einer propositionalen Struktur grundsätzlich zu kurz greifen muss. Zwar ist es korrekt, dass eine bedeutende Eigenschaft der Angst (bzw. der Furcht) in ihrer konkreten Gerichtetheit auf ein gefurchtetes Objekt, eine Eigenschaft oder ein Wahrmehmungsszenario besteht. Hier liegen dann auch Ähnlichkeiten mit Urteilsformen vor. Anders gesagt spricht nichts dagegen, dass solche objektgerichteten Zustände der Angst auch in propositionaler Form ausgedrückt werden können. Existentielle Gefühle als Stimmungen zeichnen sich im Gegensatz dazu gerade dadurch aus, dass sie überhaupt erst den Zugang zu einem solchen Erfahrungsfeld ermöglichen. Dabei handelt es sich somit nicht um einen Modus des Gefühls, der sich auf die Form "S empfindet $x$ als ein $F$ « reduzieren lässt. Denn die konkreten intentionalen Gehalte, die aufgrund existentieller Gefühle wie der Angst erschlossen werden, können vielfältig variieren, ohne dass die sie fundierende Stimmung mit den konkreten Inhalten - wie z.B. bei einem sich verändernden Wahrnehmungsfeld (mein Gang durch den dunklen Wald) - , sich gleichfalls verändern müsste. ${ }^{10}$ Das bedeutet nicht, dass existentielle Gefühle nicht auch einer nachträglichen intentionalen Erfassung zugänglich sind. Jedoch ist der ihnen korrespondierende Gehalt von anderer Art, als dies bei intentional gerichteten gegenstandsorientierten Gefühlen der Fall ist.

Wie sind jedoch die existentiellen Gefühle in ihrem Verhältnis zu ihrer leiblichen Fundierung und des sie erlebenden Subjektes genau $\mathrm{zu}$ bewerten? Sie haben zweifellos auch eine leiblich-subjektive Erfahrungsgrundlage. Dies wird besonders deutlich an basalen Gefühlen wie "sich lebendig zu fühlen" oder sich im eigenen leiblichen Vollzug als gesund, krank, müde, erfrischt oder antriebsarm zu erleben. Dass es sich bei diesen existentiellen Gefühlen gleichzeitig auch um ein präreflexives Selbsterleben des Subjekts handelt, verdeutlicht diese Passage von Thomas Fuchs aus The Ecology oft the Brain:

All conscious experience is $[\ldots]$ not only dependent on the physiological body, but emerges from the subjective body. At the most basic level, it is the body as the locus of a vague background feeling, for example, of well-being or discomfort,

\footnotetext{
${ }^{10}$ Ganz analog argumentiert Alva Noë im Hinblick auf die unterschiedlichen Verwendungsweisen von Begriffen dafür, dass sie, neben ihrer Funktion als Konstituenten von Urteilen zu fungieren, auch eine davon abweichende Funktion zur Erschließung von Erfahrungsräumen haben. Alva Noë, Concept Pluralism, Direct Perception, and the Fragility of Presence, in: Open MIND 27 (2015), 1-15.
} 
of vitality, energy, or fatigue, which may be summarized as the auto-affection of life $[\ldots]$, or the feeling of being alive. ${ }^{11}$

Es ist eine offene Forschungsfrage, ob derartige basale Gefühle, die sich zunächst einmal auf den vitalen leiblichen Zustand des Subjekts beziehen ("auto-affection of life»), überhaupt einen Weltbezug haben. ${ }^{12}$ Dieser Frage werde ich im nächsten Abschnitt aus enaktivverkörperungstheoretischer Sicht weiter nachgehen. Unbestreitbar ist, dass ein existentielles Gefühl wie die Angst mit vielfältigen leiblichen Erfahrungen einhergeht, wie die Einschnürung des Brustkorbes mit dem Gefühl der Beengung oder auch das lähmende Gefühl mit Schwindel, Atembeschwerden, Schweißausbrüche usw. Das schließt nicht aus, dass der Leib - wie am Beispiel des dunklen, bedrohlichen Waldes ausgeführt wurde - nicht auch als Medium fungieren kann, wodurch auch etwas anderes erfahren wird. Aber es hat sich bereits angedeutet, dass das Welt- und Selbstverhältnis in der Stimmung der Angst sich in einem komplexen Wechselverhältnis befindet, dass auch zu einer Störung oder partiellen Dissoziation dieses Verhältnisses führen kann. Nicht nur fundieren existentielle Gefuhle bis zu einem gewissen Grad den Zugang zu möglichen Erfahrungs- und Handlungsräumen, sondern erschweren oder verhindern diesen Zugang auch.

\section{Verkörperungstheoretische Aspekte zu Emotio- nen und existentieller Angst}

Ein wichtiger Bezugspunkt für die zeitgenössische Emotionsforschung sind die Arbeiten in den kognitiven affektiven Neurowissenschaften. Gerade Antonio Damasios Arbeiten zu Hintergrundgefühlen (background feelings) weisen eine gewisse Verwandtschaft mit dem Konzept der existentiellen Gefüle wie auch mit verkörperungstheoretischen Überlegungen zu ihrer Realisierung auf. Dies ist der Fall, obwohl Damasio die Angst gerade nicht zu den Hintergrundgefühlen zählt, sondern zu den darauf aufbauenden primären oder universellen Gefühlen rechnet. ${ }^{13}$ Dass die Abgrenzung

\footnotetext{
${ }^{11}$ Thomas Fuchs, Ecology of the Brain: The Phenomenology and Biology of the Embodied Mind, Oxford 2018, 72.

${ }^{12}$ Peter Goldie, Getting Feeling into Emotional Experience in the Right Way, in: Emotion Review 1 (2009), 232-239.

${ }^{13}$ Emilia Barile, Are Background Feelings Intentional Feelings?, in: Open Journal of Philosophy 4 (2014), 560-574, hier 562. http://dx.doi.org/10.4236/ojpp.2014.44058.
} 
hier jedoch auch für Damasio selber letztendlich nicht eindeutig ist, zeigen folgende Überlegungen zu Hintergrundgefühlen auf:

In short, certain conditions of internal state engendered by ongoing physiological processes or by the organism's interactions with the environment or both cause responses which constitute background emotions. Those emotions allow us to have, among others, the background feelings of tension or relaxation, of fatigue or energy, of well-being or malaise, of anticipation or dread. ${ }^{14}$

An dieser Passage wird deutlich, dass offenbar auch für Damasio selber nicht geklärt ist, ob bestimmte Formen der Angst nicht ebenfalls als Hintergrundgefühle fungieren (»anticipation or dread«). Doch viel wichtiger als dieses Abgrenzungsproblem ist letztendlich Damasios Zugeständnis, dass trotz der bedeutenden physiologischen Grundlagen und Prozesse Hintergrundemotionen nicht auf reine körperliche Zustände reduziert werden können, sondern eben immer auch auf Interaktionen mit der Umwelt beruhen (»engenderd by ... the organism's interactions with the environment«).

Überdies beschränkt er die Realisierung dieser Art von Gefühlen, ganz im Sinne des Verkörperungsparadigmas, nicht nur auf die neuronale Ebene, obwohl er davon ausgeht, dass jeder Basisemotion ein spezifisches neuronales Profil entspricht. Dementsprechend führt er aus, dass Hintergrundgefühle nicht so stark umweltbezogen sind wie andere Gefühle, da sie eng mit den Lebensprozessen des Leibes verbunden sind. Neben dem internen Milieu (Umweltbedingungen der Zellen im Körperinneren, wie Temperatur, Salzgehalt usw.) und viszeralen Prozesse, sieht er durchaus die Bedeutung der gesamtleiblichen Ausdruckfunktion für Gefühle wie muskuloskelletale Veränderungen, Körperhaltung und die Formierung von leiblichen Bewegungen gegeben, welche mit Hintergrundemotionen einhergehen. ${ }^{15}$

Trotzdem ist es sicherlich korrekt, dass Damasios emotionstheoretischer Ansatz im Hinblick auf seine Theorie der "somatischen Marker« im weiteren Sinne nur bedingt mit enaktiven verkörperungstheoretischen Ansätzen der Emotionen im Einklang steht. Dieser Gesichtspunkt soll im Folgenden genauer ausgeführt werden.

Die Theorie der somatischen Marker geht von der Annahme aus, dass Wahrnehmungen und Perzeptionen positiv oder negativ emoti-

\footnotetext{
${ }^{14}$ Antonio Damasio, The Feeling of What Happens: Body and Emotion in the Making of Consciousness, New York 1999, hier 32.

${ }^{15}$ Damasio, The Feeling of What Happens, hier 52-53.
} 
onal bewertet werden und es dadurch zu einer Assoziation von körperlichen Veränderungen (Schweißausbruch, Herzrasen, Verengung des Brustraums usw.) und evaluierten Handlungen kommt. Dies ist insbesondere für (prospektive) Handlungen unter Unsicherheit von Bedeutung, bei denen es aufgrund der somatischen Marker zu einer Evaluierung von Handlungsoptionen kommt. ${ }^{16}$ Letztendlich führt dieser Ansatz jedoch zu der Sichtweise, dass Hintergrundgefühle wie die existentielle verstandene Angst lediglich in der vom Gehirn vorgenommenen Repräsentation der oben angedeuteten körperlichen Prozesse bestehen. Zudem zeigt sich in der von Damasio angenommenen Funktionsweise somatischer Marker, dass er eine aus enaktiver Sicht unzutreffende Dichotomisierung von Emotionen und kognitiven Zuständen wie Denken und Wahrnehmung vornimmt. So ist es erst die innere Wahrnehmung körperlicher Zustände als Emotionen, die den zunächst nicht-affektiven und nicht evaluativen Wahrnehmungen und Gedanken ihre gefühlsmäßig bewertende Tingierung verleiht. Die Wahrnehmung des Waldes oder die herannahende Begegnung einer Menschengruppe enthalten somit zunächst nichts Bedrohliches oder Unheimliches, sondern die emotional erschlossenen Eigenschaften ( $x$ ist bedrohlich, furchterregend oder unheimlich) müssen Perzeptionen und Gedanken durch das Gehirn und die damit einhergehenden Bewertungen erst verliehen werden. Aufgrund von Erfahrungen und Habitualisierung - die auf den genannten Assoziationsmechanismen beruhen -, dürften sich im Laufe der Zeit dann Erlebnisse einstellen, bei denen die Gehalte und ihre Bewertungen nahezu instantan erlebt werden.

Aus verkörperungstheoretischer Sicht ist dieser Ansatz der kognitiv-affektiven Neurowissenschaften mit Schwierigkeiten verbunden. Wie bereits am phänomenologisch orientierten Beispiel der existentiellen Angst deutlich wurde, existiert die Angst nicht nur als gegenstandsorienter Bezug, wie die Furcht, die intentional auf ein bestimmtes Objekt oder eine Situation gerichtet ist, also einen konkreten Fokus besitzt. Vielmehr fungiert sie aufgrund ihrer existentiellen Ausrichtung auch als ein Rahmen bzw. Möglichkeitsraum der zumindest bis zu einem bestimmten Grad erst festlegt, welche Wahrnehmungen oder auch gedankliche Sinnformen überhaupt gebildet werden können.

16 Thomas Goschke/Gesine Dreisbach, Kognitiv-affektive Neurowissenschaft: Emotionale Modulation des Erinnerns, Entscheidens und Handelns, in: Klinische Psychologie \& Psychotherapie, hg. v. Hans Ulrich Wittchen/Jürgen Hoyer, Berlin/Heidelberg 2011, 129-168, hier 146. 
Wie stark dies tatsächlich nicht nur die Wahrnehmung sondern auch die gedanklich-volitionale Ebene mit betrifft, wird deutlich, wenn man sich die Wirksamkeit von mit Ängsten besetzten Zwangsstörungen (Zwangsneurosen) vergegenwärtigt. Letztere sind nicht nur mit den bekannten Symptomen, wie dem zwanghaften Händewaschens oder ähnlichen Zwangshandlungen verbunden, die mit erheblicher Angst oder Anspannungen einhergehen können. Vielmehr können Symptome derartiger Zwangssituationen auch direkt die volitionale Ebene der Entscheidungsfindung selber betreffen. ${ }^{17}$ An solchen und ähnlichen Beispielen wird deshalb deutlich, dass es nicht sinnvoll ist, die emotionale Bewertung von Wahrnehmungsszenarien oder deliberativen Prozessen als einen erst nachträglichen Vorgang in Entscheidungsprozessen bestimmen zu wollen.

Wie der Enaktivismus betont, treten Wahrnehmungen und gedanklichen Prozesse weder zeitlich noch strukturell zunächst völlig unabhängig von Gefühlen und Affektionen auf, sondern sind mit ihnen untrennbar verbunden. Bereits das basale Verhältnis von Organismus und Umwelt ist im Rahmen verkörperungstheoretischer Ansätze wie dem Enaktivismus immer schon durch eine Form der Sinngebung oder Sinnerschließung ("sense making") charakterisiert. ${ }^{18}$ Dieser aktive Prozess der Sinnerschließung ist dabei nicht als ein Vorgang zu verstehen, bei dem Eigenschaften und Relationen der Außenwelt zunächst re-präsentiert und dann einer internen emotionalen Bewertung ("appraisal«) unterzogen werden. Die Bedrohlichkeit, Unheimlichkeit oder das Furchterregende eines Wahrnehmungszenarios sind nicht einfach intrinsisch bestehende Eigenschaften, die aufgrund eines internen "Detektormechnsimus" an Perzepten festgestellt oder verworfen werden. Im Gegensatz dazu betont der Enaktivismus gerade auch im emotionstheoretischen Kontext den relationalen Charakter dieser Eigenschaften:

Objects or events need not be consciously appraised as dangers or losses to count as such; they are such, however, relative to their effects on the organism and its goals and needs. The organism, striving to maintain itself meets then world »on its own terms" and the world acquires various meanings in relation to the organism's purpose. ${ }^{19}$

\footnotetext{
${ }^{17}$ Garrit Glas, Anxiety - animal reactions and the embodiment of meaning, in: Nature and narrative: An introduction to the new philosophy of psychiatry, hg v. Bill Fulford u.a., Oxford 2003, 231-249, hier 238-239.

${ }^{18}$ Evan Thompson, Mind in life: biology, phenomenology, and the sciences of mind, Cambridge, MA 2007.

${ }_{19}$ Colombetti, The feeling body, hier 111.
} 
Im Hinblick auf diese Ausführungen stellt sich jedoch die Frage, welche Rolle eigentlich die Enkulturation für die Konstitution von Gefühlen wie der Angst hat. Sowohl Damasios Ausführungen zu den Hintergrundgefühlen als auch die verkörperungstheoretischen Ausführungen im Zitat scheinen nahe zu legen, dass es sich letztendlich um biophilosophische Ansätze zur Erklärung von Angst handelt, wenn das Verhältnis von Organismus und Umwelt zur Explikation der relationalen Konstitution von Gefühlen in den Vordergrund gestellt wird. Im Folgenden soll der Frage nachgegangen werden, welche Rolle die kulturelle Einbettung für die Entstehung existentieller Ängste im Zuge ihrer Enkulturation hat und wie dies im Rahmen einer Theorie der verkörperten Kognition weitergehend untersucht werden kann.

\section{Enkulturation als Bedingung existentieller Ängste}

So bedeutsam die organismischen und hirnphysiologischen Mechanismen zur Emergenz der (existentiellen) Angst auch sind, legt bereits das oben näher beleuchtete, paradigmatische Beispiel des "unheimlichen Waldes" als ein bedeutender Topos für existentielle Ängste in der Literatur und Filmen nahe, dass zumindest bedeutende Dimensionen der existentiellen Angst gar nicht von ihrer kulturellen Prägung, ihrer Einbettung und ihrem Aufbau getrennt werden können. Ronald de Sousa hat dafür den Begriff der "paradigmatischen Szenarios« ("paradigmatic scenarios») geprägt. Damit ist gemeint, dass aufgrund von Assoziationen Objekte oder Wahrnehmungsformationen mit bestimmten Typen von Gefühlen und Handlungsreaktionen habitualisiert werden und sich durch Geschichten, Literatur, Kunst und Filme immer stärker ausdifferenzieren. ${ }^{20}$

Wie stark die konkrete Ausdifferenzierung der Gefühle von Prozessen der Enkulturation und humanen Lebensformen insgesamt abhängt, verdeutlichen einige von Garrit Glas unterschiedenen Arten der Angst. »Enkulturation« bezieht sich in diesem Zusammenhang nicht nur auf die besondere Bedeutung, welche soziale Interaktionen und Institutionen grundsätzlich für kulturelle Lernprozesse haben. Vielmehr beinhaltet sie gerade auch die Transformation und Entwicklung der gesamten humanen Kognition (kognitiven Architektur) durch die Inkorporierung wie z.B. von symbolischen

\footnotetext{
${ }^{20}$ Ronald de Sousa, The Rationality of the Emotions, Cambridge 1987, hier 182.
} 
Kompetenzen. ${ }^{21}$ Neben der erwähnten Zwangsstörungen bei der Entscheidungsfindung, die bereits grundlegende enkulturierte Fähigkeiten wie das Vermögen zur Deliberation voraussetzt, ist es insbesondere die auf der Vulnerabilität des Menschen beruhende existentielle Angst, die in unserem Kontext aufschlussreich ist. Wie Glas ausführt, ist dieser Typus dadurch charakterisiert, dass Personen, die zum Beispiel aufgrund von Gewalterfahrungen einen starken Vertrauensverlust gegenüber ihrer Mitwelt erlitten haben, ihre Lebenswelt vielfach als unsicher, feindlich und bedrohlich wahrnehmen. Dabei sieht er diese Form der Angst in der physischen Verwundbarkeit des Menschen begründet. ${ }^{22}$

Dieser Erklärungsansatz ist sicherlich nicht unplausibel, doch geht er nicht weit genug, um das Phänomen der existentiellen Verwundbarkeit des Menschen und die daraus entspringenden existentiellen Ängste tiefergehend aufzuklären, wie auch die Anfälligkeit des Menschen für psychische Erkrankungen insgesamt. Dies leistet demgegenüber der von Thomas Fuchs geprägte Begriff der "anthropologischen Vulnerabilität". Ausgangspunkt ist in diesem $\mathrm{Zu}$ sammenhang die Frage, inwiefern die besondere Existenzform des Menschen ihn anfällig macht für Widersprüche und Grenzerfahrungen, die zu besonderen Bewältigungsstrategien oder eben auch psychischen Erkrankungen führen können. Dazu ist die von Helmut Plessner in der philosophischen Anthropologie geprägte Konzeption der »exzentrischen Positionalität" von besonderer Bedeutung. Ist das Tier in seiner konkreten sensomotorischen zentrischen Einbettung in die entsprechenden Umwelten eingepasst und gebunden, erfährt dieses fluide Tier-Umwelt Verhältnis beim Menschen eine besondere Transformation. Aufgrund seiner Fähigkeit, aus sich heraus zu treten, zu reflektieren, sich selbst aus der möglichen Perspektive anderer zu betrachten, kommt es zu einer Brechung seiner spontanen Handlungsvollzüge. ${ }^{23}$ Dies führt nicht nur aufgrund der Fähigkeit der Perspektivenübernahme dazu, dass der eigene Leib auch als beobachtbarer Körper bestimmbar wird (Plessners berühmte Formel: Körper-Haben und Leib-Sein), sondern es entstehen auch enkulturierte selbstreflexive-soziale Gefühle wie Schuld, Scham Stolz oder das Ehrgefühl. ${ }^{24}$ Zudem sind damit auch neue mögliche psychopatho-

${ }^{21}$ Merlin Donald, A mind so rare: the evolution of human consciousness, New York 2001, hier 212.

${ }^{22}$ Glas, Anxiety - animal reactions and the embodiment of meaning, hier 237.

${ }^{23}$ Helmuth Plessner, Die Stufen des Organischen und der Mensch, Berlin 1975.

${ }^{24}$ Thomas Fuchs, Anthropologische und phänomenologische Aspekte psychischer Erkrankungen, in: Psychiatrie, Psychosomatik Psychotherapie, hg. v. Hans-Jürgen Möller/Gerd Laux/Hans-Peter Kampfhammer, Berlin 2016, 417-431, hier 418. 
logische Erkrankungen wie Depressionen, Narzissmus oder auch die bereits erwähnten sozialen Phobien verbunden.

Was bedeuten diese skizzenhaften Ausführungen zur anthropologischen Vulnerabilität für das Verständnis der existentiellen Angst? Nicht nur fühlt sich der Mensch in seiner physischen Integrität besonders verletzlich, sondern das Gefühl der Bedrohung bezieht sich auf seine gesamte psychisch-soziale Existenz. Genau auf diese umfassende Art der verkörperten Lebensform des Menschen heben auch die weiteren von Glas unterschiedenen Typen der existentiellen Angst ab. So nennt er beispielsweise die Angst vor Isolation als eine weitere Variante. Sie tritt z.B. als Gefühl auf, sich wie in einem Vakuum zu befinden bzw. in einem Traum oder Nebel, ein Erlebnis, das sich bis hin zur Derealisationserfahrung steigern kann. ${ }^{25}$ Letzteres bedeutet, dass die gesamte Umgebung in solchen Zuständen als fremdartig und unvertraut erlebt wird; es besteht in solchen Situationen gegenüber der Umgebung häufig nur eine mangelnde affektive Verbundenheit. Insbesondere die existentielle Angst des Menschen vor dem eigenen Tod - als eine weitere Spielart existentieller Ängste - verdeutlicht, dass das Auftreten dieser Gefühle ein (implizites) Wissen um die eigene Endlichkeit voraussetzt. Wie schon erläutert, ist dies nur auf der Grundlage exzentrischer Positionalität überhaupt möglich.

Welchen weitergehenden Beitrag kann eine enaktive Theorie der verkörperten Kognition zur Aufklärung enkulturierter Gefühle wie der existentiellen Angst in ihren unterschiedlichen Spielarten leisten? Aufgrund der bisherigen Ausführungen ist deutlich, dass eine umfassende Erklärung darauf abzielen muss, die allgemeinen phylo- und ontogenetischen Bedingungen für die Emergenz sozialer Affektionen und Emotionen insgesamt zu erforschen. Die ist ein umfassendes Zukunftsprojekt für das erste Arbeiten vorliegen. Das Potential des Ansatzes für die konkrete Fragestellung nach der Enkulturation verkörperter existentieller Ängste soll abschließend kurz skizziert werden.

\footnotetext{
${ }^{25}$ Glas, Anxiety - animal reactions and the embodiment of meaning, hier 238.
} 


\section{Fazit und Ausblick zur weiteren Erforschung der existentiellen Angst}

Mit der Bezugnahme auf de Sousas Konzeption des "paradigmatischen Szenarios« wurde bereits deutlich, dass es vielfältige kulturelle Faktoren gibt, die dazu beitragen, Gefühle zu evozieren, zu stützen und weiter ausdifferenzieren. Zum Beispiel tragen vielfältige kulturelle Ressourcen dazu bei, wie die Inkorporation von Musik, die Gestaltung von Räumlichkeiten oder der Aufbau von verlässlichen Institutionen und Ritualen, ein existentielles Gefühl wie Vertrauen kulturell aufzubauen und zu verstetigen. Dieser Vorgang wird auch als "Scaffolding« bezeichnet. Das bedeutet in unserem Kontext, dass Akteure die Umwelt so modifizieren und neu einrichten, dass kognitive Fähigkeiten erzeugt, gestützt, vergrößert und an die gegenwärtigen und nachfolgenden Generationen vermittelt werden. ${ }^{26}$ Eine Untersuchung entsprechender Mechanismen bedeutet genau aufzuzeigen, wie z.B. kulturelle Artefakte in der Lage sind, Atmosphären und Stimmungen mit zu erzeugen, die ein Vertrauen oder eine Stimmung der Ehrfurcht wie in Kirchen erzeugen. Für die existentiellen Ängste bedeutet dies ganz analog aufzuzeigen, welche Veränderungen der Lebenswelt besonders relevant sein können, existentielles Vertrauen zu zerstören und Ängste verschiedenster Arten zu evozieren. Christoph Wulf hat beispielsweise herausgearbeitet, wie mimetische Prozesse Formen der Gewaltanwendung befördern und subjektive Erfahrung des angstbesetzten Chaos und Kontrollverlustes auslösen. ${ }^{27}$ Es ist direkt einsehbar, wie stark existentielle Erlebnisse der Angst mit solchen Prozessen verbunden sind.

Diese Ansätze zur Erforschung der Angst bedeuten nicht, existentielle Ängste lediglich auf externe kulturelle erzeugte Umweltfaktoren zurück zu führen. Die kognitiv-affektiven Neurowissenschaften tragen wichtige Erkenntnisse zum Auftreten existentieller Ängste bei. Doch handelt es sich um ein Gesamtphänomen, bei dem leibliche, psychische und kulturelle Faktoren in ihrem Zusammenspiel untersucht werden müssen. Auch für die eingangs gestellte Frage nach der Ausrichtung von Gefühlen enthält dieser Befund einen wichtigen Hinweis: Gefühle beziehen sich immer auf das wechsel-

\footnotetext{
${ }^{26}$ Giovanna Colombetti/Joel Krueger, Scaffoldings of the affective mind, in: Philosophical Psychology 28(8) (2015), 1157-1176, hier 1157.

${ }^{27}$ Christoph Wulf, The Creation of Body Knowledge in Mimetic Processes, in: Embodiment in Evolution and Culture, hg. v. Gregor Etzelmüller/Christian Tewes, Tübingen 2016, 249-264, hier 252.
} 
seitige Zusammenspiel von Selbst- und Welterfahrungen in ihren unterschiedlichen Formen derVerkörperung.

- PD Dr. Christian Tewes ist Privatdozent für Philosophie an der Friedrich-SchillerUniversität Jena. Zu seinen Forschungsgebieten zählen die Philosophie des Geistes, Anthropologie, Phänomenologie und die Theorie der Verkörperten Kognition (besonders der Enaktivismus). 